\title{
Isolated tricuspid valve infective endocarditis in young drug abusers
}

\author{
Cenap Ozkara ${ }^{1}$, Omer Faruk Dogan ${ }^{2}$, Cevdet Furat ${ }^{3}$ \\ ${ }^{1}$ Department of Cardiovascular Surgery, Corlu Sifa Hospital, Istanbul, Turkey \\ ${ }^{2}$ Department of Cardiovascular Surgery, Adana Numune Education and Training Hospital, Adana, Turkey \\ ${ }^{3}$ Zonguldak Ereğli Devlet Hastanesi, Zonguldak, Turkey \\ Email: ofdogan@hacettepe.edu.tr
}

Received 29 March 2012; revised 26 April 2012; accepted 20 May 2012

\begin{abstract}
Isolated tricuspid valve infective endocarditis (TVIE) is a rare clinical condition. Thus, there is no common consensus for the treatment options for TVIE. Vegetectomy and valvulectomy, valve repair, and valve replacement, which are controversial in regard to hemodynamic consequences in right-sided low-pressure system and long-term prognosis. We present 2 young intravenous drug users with TVIE and our surgical strategy.
\end{abstract}

Keywords: Infective Endocarditis; Tricuspid Valve Replacement; Intravenous Drugs

\section{INTRODUCTION}

Tricuspid valve infective endocarditis is rare and accounts for $5 \%$ of infective endocarditis [1]. Its causes are insertion of central venous catheter, placement of a pacemaker, a history of intravenous drug use, and haemodialysis. The reasons why TVIE is rare are thought to be the tricuspid and pulmonary valves are not strained because of low pressure; and oxygen saturation is low. Nevertheless, reports of TVIE are growing because of the increasing frequency of drug-user patients [2]. Pulmonary embolism occurs in $75 \%$ to $100 \%$ of TVIE patients [3]. Although TVIE is successfully treated medically in about $75 \%$ of patients, conservative treatment is not always effective, and surgical treatment is required in approximately the remaining $25 \%$ [1]. We report on 2 surgical cases who are drug users with TVIE.

\section{CASE REPORTS}

\subsection{Case 1}

A 17-year-old boy was referred to our emergency unit with the complaint of high fever, dispnea, and palpitation for about a week. Echocardiography showed severe tricuspid regurgitation (TR) and vegetation on the tricuspid valve. He was diagnosed with isolated TVIE and underwent medical treatment in the emergency unit. Physical examination has shown that there was abdominal distention. Pulmonary oscultation has shown that there was a ral and roncus. Blood tests showed an inflammatory reaction with WBC count $28,400 / \mu 1$ and CRP level 9.25 $\mathrm{mg} / \mathrm{dl}$. Echocardiographic examinations showed a TR grade of 3 - 4 and mobile vegetation, $43 \times 27 \mathrm{~mm}$, on the tricuspid valve (Figure 1). Right atrial and ventricular dilatation was seen. Chest CT demonstrated multiple infiltrates in both lung fields and multiple pulmonary embolisms. He underwent sequential medical treatment in our hospital. The patient hospitalised in intensive care unit. During the medical treatment the size of the vegetation has decreased $2.3 \mathrm{~mm}$. However, arterial blood gas analyses have showed that the saturation of the arterial blood $85 \%-90 \%$. 4 weeks after medical treatment, he underwent surgery in the active stage because of repeated pulmonary embolism during medical treatment. The operation was performed in the standard manner, on arrest with $\mathrm{CPB}$. When the right atrium was opened, a large vegetation was found attached to the anterior leaflet of the tricuspid valve. The septal leaflet was detached from the annulus. Because the infection involved all leaflets, a total resection of the tricuspid valve was performed. The tricuspid valve replacement (TVR) was performed by using a $33 \mathrm{~mm}$ Carpentier-Edwards pericardial bioprosthesis (Edwards Lifesciences, Irvine, CA). His postoperative course was uneventful. Medical treatment and antibiotic regimens have continued postoperatively. The patient discharged home with a well clinical condition. About 3 months after surgery, he remains well with no recurrence of endocarditis.

\subsection{Case 2}

A 22-year-old intravenous drug user man was referred to our hospital with a history of fever, early fatigue and dispnea. He also complaintd of cough, and appetite loss for about 1 week. Echocardiography showed TR and 


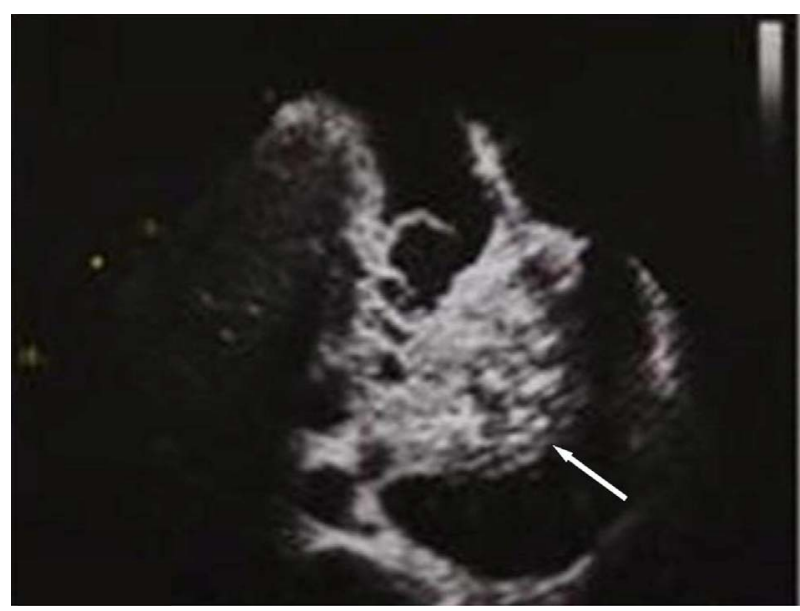

Figure 1. The echocardiographic examinations shows severe tricuspid regurgitation and mobile vegetation (white arrow). The size of the vegetation is $43 \times 27 \mathrm{~mm}$ on the tricuspid valve.

vegetation on the tricuspid valve. Isolated TVIE was diagnosed, and he underwent medical treatment. Physical examination was normal. Blood tests showed a inflammatory reaction with a WBC count of $16,500 / \mu 1$ and a CRP level of $5.35 \mathrm{mg} / \mathrm{dl}$. Echocardiography showed TR grade 4 and mobile vegetation, $24 \times 11 \mathrm{~mm}$, on the tricuspid valve. There was a perforation on the anterior leaflet of the tricuspid valve. Chest CT demonstrated multiple infiltrates and cavitations in both lung fields and multiple pulmonary embolisms (Figure 2). 2 weeks after medical treatment he underwent an operation in the healed stage.

Operation was performed in the standard manner, on arrest with CPB. When the right atrium was opened, multiple vegetations were found attached to the anterior, septal leaflet of the tricuspid valve. Torn chordae were seen in the operation. Because the infection was generalized, and there was a valvular perforation a total resection of the tricuspid valve with its vegetation was performed. No.33 Carpentier-Edwards pericardial bioprosthesis (Edwards Lifesciences, Irvine, CA) was replaced. His postoperative course was uneventful. He remains well about 2 months after surgery with no recurrence of endocarditis.

\section{DISCUSSION}

The treatment of TVIE likes to left-sided endocarditis. After the diagnosis of TVIE, medical treatment with antibiotics is indicated. If the patients' status is possible, medical management should be continued until signs of infection disappear for 4 to 6 weeks. The operative indications for TVIE in the active stage are severe tricuspid regurgitation, septic embolus, recurrent pulmonary embolism, and the presence of mobile vegetation of more than $10 \mathrm{~mm}$ [1]. In our both patients, early surgical ap-

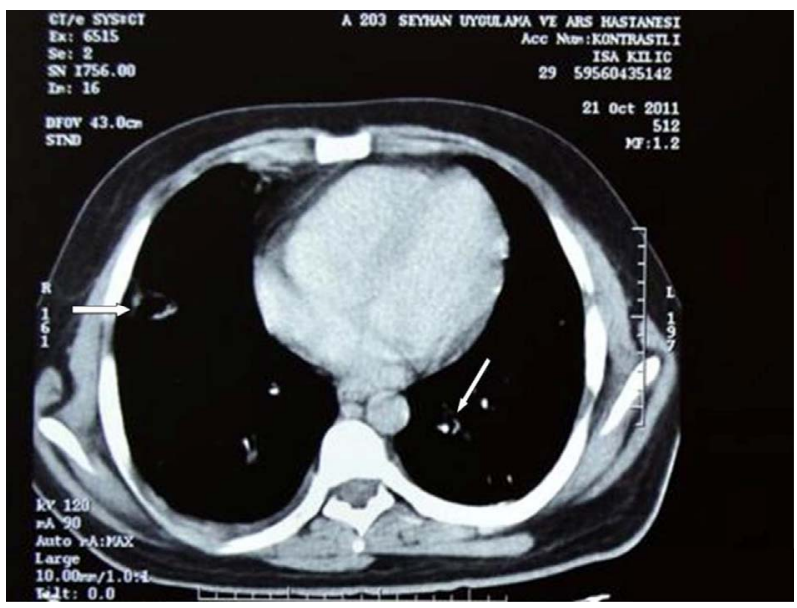

Figure 2. Chest computed tomography demonstrates multiple infiltrations and cavitations in both lung due to multiple pulmonary embolisms (white arrows).

proach was indicated because of septic recurrent pulmonary emboli from vegetations during the active stage.

In surgical treatment for TVIE, a complete debridement of infected tissue including the tricuspid valve and a reduction of tricuspid regurgitation are important to prevent the recurrence of endocarditis and right ventricular dysfunction after surgery.

In the literature, the surgical options are vegetectomy and valvulectomy [2], valve repair [3-5] and valve replacement [6]. Vegetectomy and valvulectomy have the benefit of using no prosthetic material, but they result in right ventricular dysfunction because of massive TR after surgery and require reoperation in more than $20 \%$ of patients [6,7]. Lange et co-workers indicated that valvuloplasty for their patients of TVIE resulted in good outcomes in the follow-up period of $29 \pm 18$ months after surgery [4]. Konstantinov published a case of total resection and complete reconstruction of all the leaflets of the tricuspid valve with autologous pericardium and artificial chordae in acute infective endocarditis [8].

A limited number of medical reports with regard to the outcome of valve replacement for TVIE has been found. Tokunaga et al. reported excellent long-term outcomes with isolated TVR in 31 patients with tricuspid valve disease whose operative mortality was $6.5 \%$ [9]. A partial replacement using a mitral homograft has been reported by Couetil et al. [10]. Morokuma et al. have reported the three cases with isolated TVIE and the surgical results. They used the bioprosthetic valve in one of these three patients [6].

For optimal treatment in TVIE patients there are many of questions are waited the responces. The main question is: Are the mechanical or biologic valves better in regard to thrombosis or durability in the right-sided low pressure system? This question is controversial yet and is not being completely answered. 
Although, it is standard procedure that operations are performed under $\mathrm{CPB}$, some patients with TVIE cannot tolerate it because of severe lung injury as a result of pulmonary embolism. In our two patients, valvular repair was not possible. Thus, we chosed bioprosthetic tricuspid valve replacement for the tratment of TVIE. There is no side effect of the biologic valves during the follow-up period in both patients. If it is possible tricuspid valve repair is now the choice ofsurgical option for patients with TVIE. However, we believe that if tricuspid destruction is severe and not suitable for valve repair due to infection involves all three leaflets and the subvalvular apparatus tricuspid valve replacement is indicated.

\section{CONCLUSION}

We report 2 rare surgical cases of isolated TVIE in young drug users and clinical outcomes. We believe that tricuspid bioprosthetic valve replacement can be done safely for a surgical option in patients TVIE with regard to freedom of recurrence of endocarditis and valvular competence.

\section{REFERENCES}

[1] Chan, P., Ogilby, J.D. and Segal, B. (1989) Tricuspid valve endocarditis. American Heart Journal, 117, 11401146. doi:10.1016/0002-8703(89)90874-0

[2] Arbulu, A., Holmes, R.J. and Asfaw, I. (1991) Tricuspid valvulectomy without replacement. Twenty years' experience. Journal of Thoracic and Cardiovascular Surgery, 102, 917-922.

[3] Allen, M.D., Slachman, F., Eddy, A.C., Cohen, D., Otto,
C.M. and Pearlman, A.S. (1991) Tricuspid valve repair for tricuspid valve endocarditis: Tricuspid valve "recycling". The Annals of Thoracic Surgery, 51, 593-598. doi:10.1016/0003-4975(91)90317-J

[4] Lange, R., De Simone, R., Bauernschmitt, R., Tanzeem, A., Schmidt, C. and Hagl, S. (1996) Tricuspid valve reconstruction: A treatment option in acute endocarditis. European Journal Cardio-Thoracic Surgery, 10, 320-326. doi:10.1016/S1010-7940(96)80089-3

[5] Gottardi, R., Bialy, J., Devyatko, E., Tschernich, H., Czerny, M., et al. (2007) Midterm follow-up of tricuspid valve reconstruction due to active infective endocarditis. The Annals of Thoracic Surgery, 84, 1943-1949. doi:10.1016/j.athoracsur.2007.04.116

[6] Morokuma, H., Minato, N., Kamohara, K. and Minematsu, N. (2010) Three surgical cases of isolated tricuspid valve infective endocarditis. Annals of Thoracic and Cardiovascular Surgery, 16, 134-138.

[7] Robbins, M.J., Soeiro, R., Frishman, W.H. and Strom, J.A. (1986) Right-sided valvular endocarditis: Etiology, diagnosis, and an approach to therapy. American Heart Journal, 111, 128-135. doi:10.1016/0002-8703(86)90564-8

[8] Konstantinov, I.E. (2008) Total resection and complete reconstruction of the tricuspid valve in acute infective endocarditis. Journal of Thoracic and Cardiovascular Surgery, 136, 531-532. doi:10.1016/j.jtcvs.2007.12.061

[9] Tokunaga, S., Masuda, M., Shiose, A., Tomita, Y., Morita, S., et al. (2008) Long-term results of isolated tricuspid valve replacement. Asian Cardiovascular \& Thoracic Annals, 16, 25-28.

[10] Couetil, J.P., Argyriadis, P.G., Shafy, A., Cohen, A., Berrebi, A.J., et al. (2002) Partial replacement of the tricuspid valve by mitral homografts in acute endocarditis. The Annals of Thoracic Surgery, 73, 1808-1812. doi:10.1016/S0003-4975(02)03574-9 